\title{
Glosario de términos usados en riego por goteo y su traducción al inglés (A Glossary of Drip Irrigation Terms and Their Translations in English) ${ }^{1}$
}

\author{
Tatiana Sanchez, Eric Simonne, y Monica Ozores-Hampton²
}

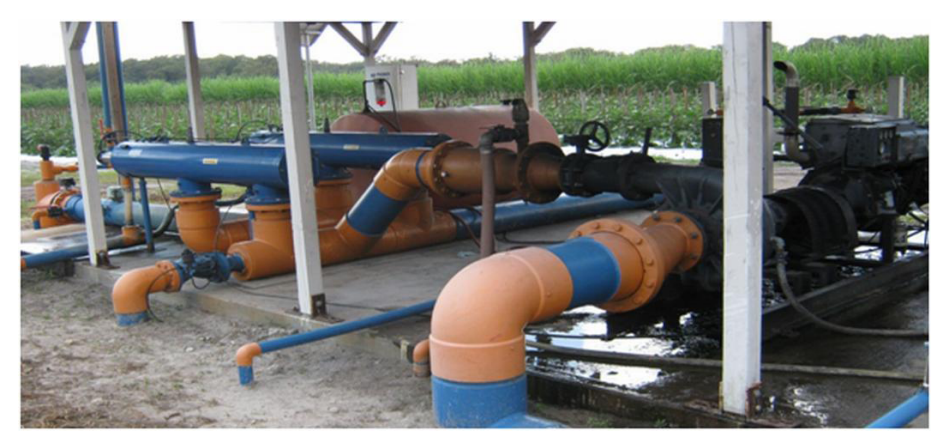

Figure 1. Vista panorámica de un cabezal central de riego por goteo/ Panoramic view of a drip irrigation pump station.

Credits: UF/IFAS File Photo

En esta publicación se presenta el léxico técnico de riego por goteo en dos secciones. En la primera sección se describen en orden alfabético los términos y sus definiciones en español. En la segunda sección se enlista de forma alfabética los términos y sus definiciones en inglés. En ambas secciones, cada término incluye entre paréntesis su respectiva traducción al inglés o español. Las unidades son expresadas de acuerdo al sistema métrico y al sistema inglés.

This publication includes the drip irrigation lexicon in two sections. In the first section, terms and their definitions are alphabetically described in Spanish. The second section lists terms and their definitions in English in alphabetical order. In both sections, each term includes its respective translation into English or Spanish in parenthesis. Units are expressed according to the English and metric systems.

\section{Sección 1. Léxico técnico de riego por goteo}

Accesorios (Fittings): Conjunto de conexiones y dispositivos de cierre o sellado usados para construir un sistema de riego por goteo, que incluyen conectores, T's, codos, tapones y tapones terminales. Los accesorios pueden ser de diferentes tipos, incluyendo series de compresión, dentadas $\mathrm{y}$ de roscas (de tuerca o anillo).

Acidificación (Acidification): Adición de un ácido-tales como ácido fosfórico, sulfúrico o clorhídrico (muriático)en un sistema de irrigación. Esta práctica se lleva a cabo en mantenimiento para mejorar la efectividad de la cloración.

Ácido (Acid): Compuesto que libera iones $\mathrm{H}^{+}$cuando se encuentra en disolución. Compuestos tales como ácido clorhídrico $(\mathrm{HCl})$ o ácido acético $\left(\mathrm{CH}_{3}-\mathrm{COOH}\right)$ son ejemplos de ácidos.

1. Este documento, HS1192, es uno de una serie de publicaciones del Departamento de Ciencias Hortícolas, Servicio de Extensión Cooperativa de la Florida, Instituto de Alimentos y Ciencias Agrícolas, Universidad de la Florida (UF/IFAS Extension). Fecha de primera publicación: julio 2011. Repasado octubre 2017 y abril 2021. Visite nuestro sitio web EDIS en https://edis.ifas.ufl.edu.

2. Tatiana Sanchez, commercial horticulture agent, UF/IFAS Extension Alachua County; Eric Simonne, professor, Horticultural Sciences Department, and director, Northeast District Extension; and Monica Ozores-Hampton, former associate professor, Horticultural Sciences Department; UF/IFAS Extension, Gainesville, FL 32611. 
Ácido Clorhídrico (HCl) (Hydrochloric acid): Ácido usado frecuentemente para bajar el $\mathrm{pH}$ del agua e incrementar la eficiencia de la cloración. El uso de $\mathrm{HCl}$ está prohibido en la producción orgánica certificada en los EEUU.

Ácido débil (Weak acid): Ácido que se disocia parcialmente en agua. Los ácidos débiles comunes son ácido fosfórico $\left(\mathrm{H}_{3} \mathrm{PO}_{4}\right)$, ácido bórico $\left(\mathrm{H}_{3} \mathrm{BO}_{3}\right)$, ácido acético $\left(\mathrm{CH}_{3}-\mathrm{COOH}\right)$ y ácido cítrico $\left[\mathrm{COOH}-\mathrm{CH}_{2}-(\mathrm{COOH}-\mathrm{C}-\mathrm{OH})-\mathrm{CH}_{2}-\mathrm{COOH}\right]$.

Ácido Fosfórico $\left(\mathrm{H}_{3} \mathrm{PO}_{4}\right)$ (Phosphoric acid): Ácido usado frecuentemente para bajar el $\mathrm{pH}$ del agua e incrementar la eficiencia de la cloración. El uso de ácido fosfórico está prohibido en la producción orgánica certificada.

Ácido fuerte (Strong acid): Ácido fuerte que se disocia totalmente en agua. Los ácidos fuertes comunes son ácido clorhídrico $(\mathrm{HCl})$ y ácido sulfúrico $\left(\mathrm{H}_{2} \mathrm{SO}_{4}\right)$.

Ácido hipocloroso (HOCl) (Hypochlorous acid): Ácido débil generado mediante la cloración de productos. El ácido hipocloroso destruye la materia orgánica. El uso de $\mathrm{HOCl}$ está restringido en la producción orgánica.

Ácido muriático (Muriatic acid): Nombre con el que se conoce también al ácido clorhídrico $(\mathrm{HCl})$.

Ácido peracético (ácido conocido también como Peroxiacético) (Peracetic acid, also known as peroxyacetic): Mezcla de ácido acético $\left(\mathrm{CH}_{3}-\mathrm{COOH}\right)$ y peróxido de hidrógeno $\left(\mathrm{H}_{2} \mathrm{O}_{2}\right)$ en una solución acuosa que puede ser usada en la producción orgánica certificada como substituto de productos de cloración.

Ácido sulfúrico $\left(\mathrm{H}_{2} \mathrm{SO}_{4}\right)$ (Sulfuric acid): Ácido usado frecuentemente para bajar el $\mathrm{pH}$ del agua para incrementar la eficiencia de la cloración. El uso del ácido sulfúrico está prohibido en la producción orgánica certificada.

Agencia de certificación (Certifying agency): Organismo tercero independiente y acreditado que verifica que una operación orgánica certificada cumpla con las regulaciones descritas en los Estándares Nacionales de Producción Orgánica asegurándose de que sea apropiada para su sistema de agricultura.

Agente de limpieza (Cleaning agent): Substancia usada para remover suciedad, residuos y contaminantes.
Alcalinidad del agua (Water alkalinity): Habilidad del agua para neutralizar ácidos. La alcalinidad del agua se basa en el contenido de iones de hidróxido $\left(\mathrm{OH}^{-}\right)$, carbonato $\left(\mathrm{CO}_{3}^{2-}\right)$ y bicarbonato $\left(\mathrm{HCO}_{3}^{-}\right)$.

Alguicida (Algicide): Substancia tóxica a las algas.

Azufre en polvo (Sulfur, powdered): Uso de azufre elemental (S) en forma de polvo amarillo está permitido en la producción orgánica certificada. Es comúnmente utilizado para disminuir el $\mathrm{pH}$ del suelo, pero requiere de la intervención de microorganismos del suelo para su conversión química.

Bactericida (Bactericide): Substancia que mata bacterias.

Base (Base): Compuesto que produce iones $\mathrm{OH}^{-}$cuando se encuentra en disolución. Compuestos tales como hidróxido de potasio $(\mathrm{KOH})$ o hidróxido de sodio $(\mathrm{NaOH})$ son ejemplos de bases.

Buenas Prácticas de Manejo (BPM) (Best Management Practices, BMP): Conjunto de prácticas culturales utilizadas para incrementar la eficiencia del programa de riego y fertilización mientras se minimiza el impacto ambiental de la producción.

Capacidad de campo (Field capacity): Contenido de humedad en el suelo después de que el agua libre ha sido drenada por gravedad.

Caudal (Flow rate): Cantidad de agua que circula en las tuberías en un periodo de tiempo dado. Para microirrigación (riego por goteo), el caudal es expresado en $\mathrm{L} / \mathrm{h}$ o $\mathrm{L} / \mathrm{min}[\mathrm{gal} / \mathrm{h}$ (gph) o en gal/min (gpm)].

Caudal de goteo (Application rate): Tasa promedio de la aplicación de agua a través de un sistema de irrigación. Para riego por goteo, el caudal de goteo es expresado en $\mathrm{L} / \mathrm{h} / \mathrm{m}$ o $\mathrm{L} / \mathrm{min} / \mathrm{emisor}$ (gal/h/100 ft o gal/min/emisor).

Caudal real de riego (Water applied): Cantidad real de agua aplicada durante un ciclo de riego. Para riego por goteo, ésta es expresada en $\mathrm{L} / \mathrm{m}$ de cinta de goteo (gal/100 pies de cinta de goteo).

Cloración (Chlorination): Incorporación de cloro-a una dosis calculada - al sistema de irrigación. Para la cloración se puede usar hipoclorito de sodio líquido (blanqueador casero) o gas de cloro. Algunos agentes de cloración son permitidos en la producción orgánica certificada. 
Conector cinta-cinta (Tape-to-tape connector): Pieza utilizada para reparar o reemplazar una sección de cinta de goteo con problemas de fugas de agua. El conector cintacinta permite la unión de dos cintas de goteo.

Conector cinta-lateral (Tape-to-lateral connector): Pieza también conocida como adaptador dentado que es colocada al final de la cinta de goteo (terminal de rosca) para unirla con la línea lateral (terminal insertable).

Detergente (Detergent): Substancia sintética (que no es jabón) usada para cambiar la tensión superficial, eliminando el aceite, la grasa y otras substancias relativamente insolubles en agua. Los detergentes no están permitidos en la producción orgánica certificada.

Dispositivo anti-sifón (Anti-siphon device) (ver dispositivo de prevención de retro-flujo): Dispositivo de seguridad utilizado para prevenir el retro-flujo de agua de irrigación a la fuente de abastecimiento ocasionado por retro-sifonaje.

Dispositivo de prevención de retro-flujo (Backflow-prevention device) (ver dispositivo anti-sifón): Dispositivo requerido por la ley en la Florida que previene el retorno de agua contaminada a la fuente de abastecimiento de agua, en caso de ocurrir una situación de retro-flujo.

Dureza del agua (Water hardness): Suma de iones polivalentes -tales como calcio, magnesio, aluminio, o hierro- en solución. La dureza es expresada como carbonato de calcio equivalente en $\mathrm{mg} / \mathrm{L}$ o ppm, y los valores son usados para clasificar el agua como blanda (0-20 mg/L), moderadamente blanda (20-40 mg/L), ligeramente dura (40-60 $\mathrm{mg} / \mathrm{L})$, moderadamente dura (60-80 mg/L), dura (80-120 $\mathrm{mg} / \mathrm{L})$ o muy dura (>120 mg/L).

Eficiencia de aplicación (Application efficiency): Porcentaje de agua aplicada a través de un sistema de irrigación que es almacenada en la zona radicular y está disponible para uso del cultivo.

Emisor o gotero (Emitter): Dispositivo de entrega o apertura en una tubería de micro-irrigación que regula la aplicación de agua. Un emisor crea un flujo controlado expresado en $\mathrm{L} / \mathrm{min} /$ emisor o $\mathrm{L} / \mathrm{m} / \mathrm{h}$ (gal $/ \mathrm{min} /$ emisor o gal/100 pies/h).

Emisor o gotero auto-compensante de presión (Pressurecompensating emitter): Emisor diseñado para mantener una descarga constante (flujo) dentro de un rango de presión de operación y elevación.
Emisor o gotero de flujo turbulento (Turbulent-flow emitter): Emisor con una serie de canales que aceleran el flujo de agua y, por lo tanto, previenen el asentamiento de partículas y taponamiento de los emisores.

Emisor o gotero sensible a la presión (Pressure-sensitive emitter): Emisor que libera gran cantidad de agua a presiones altas y una menor cantidad de agua a presiones bajas, lo cual comúnmente ocurre en las líneas laterales largas o en superficies irregulares.

Evapotranspiración (ET) (Evapotranspiration): Pérdida de agua que resulta de la combinación de lo que se evapora desde el suelo y de la transpiración de las plantas.

Fertirrigación (Fertigation): Aplicación de fertilizantes solubles (nutrientes vegetales) a través de un sistema de riego por goteo. La fertirrigación está permitida en sistemas de producción orgánica certificada siempre que las fuentes de fertilizantes usadas sean permitidas por los estándares del Programa Nacional de Agricultura Orgánica (NOP).

Filtro (Filter): Dispositivo cilíndrico que puede contener una malla o serie de discos de un específico mesh (número de orificios por pulgada cuadrada de malla) o bien puede ser llenado de arena sólida mediana, diseñado para atrapar las partículas sólidas suficientemente grandes como para obstruir los emisores.

Filtro de arena (Media filter): Tanque presurizado rellenado de arena y grava fina. La arena es colocada por encima de la grava. Las arenas de corte filoso o rocas trituradas son más eficientes que las arenas redondas para capturar los tejidos suaves de algas. Los filtros de arena deberían ser usados para filtrar el agua que contiene altos niveles de materia orgánica.

Filtro de discos (Disk filter): Conjunto de discos apilados, redondos y con ranuras que son usados para filtrar el agua en un sistema de riego por goteo. A menor tamaño de las ranuras en los discos, mayor cantidad de agua filtrada. Cada disco tiene ranuras en ambos lados. Los sedimentos y la materia orgánica son acumulados sobre los discos a medida que el agua circula en las ranuras. Los discos son reusables. Una vez separados, ellos pueden ser fácilmente limpiados usando agua a presión y/o una solución limpiadora.

Filtro de malla (Screen filter): Tipo de filtro que utiliza una malla rígida para separar arenas y otras partículas del agua de riego. 
Golpe de ariete (Water hammer): Aumento repentino de la presión que ocurre cuando hay un cierre, una interrupción brusca del flujo o debido a un cambio de dirección del mismo. El golpe de ariete puede ser limitado o evitado si se permite la entrada gradual del agua al sistema o mediante un diseño del sistema de irrigación que contemple una velocidad del agua menor a $1.5 \mathrm{~m} / \mathrm{seg}$ (5 pies/seg).

\section{Instituto de Revisión de Materiales Orgánicos (Organic} Materials Review Institute, OMRI): Organización nacional no lucrativa que determina cuales insumos cumplen con los Estándares del Programa Nacional Orgánico (OMRI, http://www.omri.org). El uso de los productos enlistados por el OMRI requiere de la aprobación de una agencia de certificación. La lista de productos presentada por la OMRI no es garantía de la eficacia de los productos enlistados.

\section{Inyector Mazzei (Mazzei injector) (ver Inyector tipo} Venturi): Inyector patentado tipo Venturi, en forma de T, que no incluye partes móviles.

Inyector tipoVenturi (Venturi injector): Dispositivo que en su interior tiene un estrechamiento gradual que opera bajo el principio de que una disminución de presión es acompañada de un cambio en la velocidad del flujo de agua al pasar por el estrechamiento. La disminución de presión al interior del injector tipo venturi debe ser suficiente como para crear una presión negativa (vacío) en relación a la presión atmosférica. Bajo estas condiciones, el fluido almacenado en el tanque será succionado hacia el inyector.

Jabón (Soap): Sales alcalinas de ácidos grasos usados para remover partículas hidrofílicas.

Línea lateral (Lateral line): Línea real de goteo. Son mangueras sobre o dentro de las cuales son colocados o insertados los goteros.

Línea principal (Mainline): Tubería usada en el sistema de riego por goteo para conducir agua desde la fuente de abastecimiento hacia las líneas secundarias. Puede estar hecha de material de PVC rígido o polietileno suave con diferentes diámetros desde 1.25 a $10 \mathrm{~cm}$ (0.5 a 4 pulgadas).

\section{Línea secundaria (Sub-mainline or Secondary line):}

Tubería usada para distribuir agua a un cierto número de líneas laterales.

Medidor de flujo (Flow meter): Dispositivo usado para medir caudales de flujo en un sistema de riego durante el curso de un ciclo de cultivo.
Micro-irrigación (Micro-irrigation): Sinónimo de riego por goteo.

Partes por millón (ppm) (Part-per-million): Proporción de uno en un millón: 1 ppm $=1 / 1,000,000$. Una medida de ppm también puede representar concentraciones: $1 \mathrm{ppm}=$ $1 \mathrm{mg} / \mathrm{L} ; 1 \%=10,000 \mathrm{ppm}$.

Pérdida de presión (Pressure loss): Pérdida de presión del agua en condiciones de flujo causada por impurezas en el filtro, fricción en las tuberías y otras partes, y cambios en elevación.

Perforador (Hole punch): Dispositivo con el que se hacen los orificios redondos en las tuberías para conectar la cinta de goteo a los laterales (disponible en varios diámetros).

pH (pH): Parámetro que representa la cantidad de acidez (concentración de iones $\mathrm{H}^{+}$) en solución, representado por un número entre 0 y 14 que se calcula como $\mathrm{pH}=-\log$ $\left[\mathrm{H}^{+}\right]$. El pH puede medirse simplemente con un medidor de $\mathrm{pH}$ (pH-metro). La solución es ácida cuando el $\mathrm{pH}<7$, neutra cuando el $\mathrm{pH}=7$, y alcalina cuando el $\mathrm{pH}>7$. El pH afecta la solubilidad y las formas iónicas en solución. El pH es el parámetro químico simple más importante para agua y suelo.

Presión (Pressure): La "fuerza" que propulsa el agua a través de las tuberías. La presión estática (no flujo) común en sistemas de irrigación es de 1.4-4.8 bar [20-70 lb/ pulgada $\left.^{2}(\mathrm{psi})\right]$. Los sistemas de irrigación operan bajo presión hídrica dinámica (flujo), la cual es reducida por los aumentos en elevación y las pérdidas por fricción causadas por el contacto del agua en las paredes de las tuberías.

Presión nominal (Pressure rating): Máxima presión que una tubería u otro componente del sistema de riego por goteo puede resistir sin fallas. Por ejemplo, para la clase de tubería plástica 160 se especifica una presión máxima de 11.0 bar $\left[160 \mathrm{lb} /\right.$ pulgada $^{2}$ (psi)]. La tubería de aluminio tiene una presión máxima de 10.0-10.3 bar (145-150 psi).

Presión por gravedad (en lb/pulgada ${ }^{2}$ o psi) (Pressure due to gravity, in pounds-per-square-inch or psi): Esta medida puede ser calculada como la ganancia (pendiente cuesta abajo) o perdida (pendiente cuesta arriba) al multiplicar la altura de la columna de agua en $\mathrm{m}$ por $0.0979 \mathrm{bar} / \mathrm{m}$ (en pies por $0.433 \mathrm{psi} / \mathrm{pie}$ ). Por ejemplo, si una cinta de riego por goteo de $60 \mathrm{~m}$ (200 pies) es colocada sobre el suelo con una pendiente cuesta abajo de $0.9 \mathrm{~m} / 30 \mathrm{~m}$ (3\%) [3 pies/100 pies (3\%)], la ganancia de presión debido a la gravedad será de $60 \times 0.03 \times 0.0979=0.18$ bar $(200 \times 0.03 \times 0.433=2.59$ psi). 
Programa Nacional de Producción Orgánica (National Organic Program, NOP): Programa federal creado como resultado del Acta de Producción de Alimentos Orgánicos de 1990 (titulo IX de la iniciativa de Ley del Campo) y operado bajo el Servicio de Comercialización Agrícola del Departamento de Agricultura de los Estados Unidos (http://www.ams.usda.gov/nop/indexIE.htm).

Programación del riego (Irrigation schedule): Plan de riego y procedimientos que determinan el momento y la frecuencia de un evento de riego.

\section{Punto de marchitez permanente (Permanent wilting} point): Contenido de humedad en el suelo, particularmente en la zona radicular, cuando la planta no puede extraer más agua del suelo.

Quelato (Chelate): Compuesto que protege metales polivalentes en dos o más sitios de intercambio catiónicos. El quelato es con frecuencia un componente que está en formulaciones listas para ser usadas en limpieza de riego por goteo. El uso de quelatos sintéticos no está permitido en la producción orgánica certificada para limpieza de sistemas de riego por goteo, sin embargo los quelatos sintéticos pueden ser usados en la producción orgánica certificada para corregir una deficiencia de micronutrientes debidamente documentada.

Quimirrigación (Chemigation): Término general que se refiere a la aplicación de químicos solubles en agua a través de un sistema de riego. La quimirrigación incluye (cuando es permisible) la aplicación de fertilizantes, ácidos, cloro y pesticidas.

Regulador de presión (Pressure regulator): Dispositivo que reduce y mantiene la presión del agua entrante al sistema de riego de baja presión. La presión típica de agua doméstica es de hasta 3.4-4.1 bar (50-60 psi), mientras que los sistemas de riego por goteo están diseñados para no exceder de 0.5-0.8 bar (8-12 psi) en la cinta de goteo. Debido a pérdidas por fricción, la presión en las tuberías de suministro puede ser de 1.4-2.0 bar (20-30 psi), por lo que se requiere un regulador de presión. Las medidas importantes de un regulador de presión son el diámetro, la presión de la corriente, y el flujo máximo permitido por el regulador de presión.

Riego por goteo (Drip irrigation, Trickle irrigation): Método de irrigación que utiliza la aplicación lenta de agua bajo baja presión a través de aperturas o dispositivos sujetados justo encima, a ras o debajo de la superficie del suelo.
Separación entre emisores (Emitter spacing): Distancia entre dos emisores consecutivos. Las separaciones típicas entre emisores para cultivos hortícolas son 10, 20, $30 \mathrm{~cm} \mathrm{(4,}$ 8 y 12 pulgadas) y para arándano es de $45 \mathrm{~cm}$ (18 pulgadas).

Separador de arena (Sand separator): Dispositivo llamado también hidrociclón que utiliza la fuerza centrífuga para separar arenas y otras partículas pesadas del agua de riego. No es un filtro verdadero sino más bien puede ser considerado como un dispositivo pre-filtro.

\section{Sistema de Información electrónica de base de datos (Electronic Database Information System, EDIS): Base de datos en línea donde las recomendaciones científicas de la Universidad de la Florida, se encuentran revisadas, actualizadas y disponibles (https://edis.ifas.ufl.edu).}

Sobre-fertirrigar (Overfertigating): Aplicación de fertilizantes a través del riego que supera la dosis recomendada sobre un determinado cultivo. Sobre-fertirrigar puede resultar en la lixiviación de nutrientes por debajo de la zona de la raíz.

Sobre-regar (Overwatering): Aplicación de agua en cantidad que sobrepasa las necesidades requeridas por el cultivo o que excede la capacidad de retención de humedad del suelo. Sobre-regar puede resultar en la lixiviación de nutrientes por debajo de la zona de la raíz.

Tapón (Goof plug): Artefacto insertable usado para cerrar orificios en la tubería principal y microtubos donde ciertos dispositivos han sido removidos o no son requeridos. También puede ser utilizado para tapar algún orificio que ha sido hecho accidentalmente.

Tapón de fin de línea con purga automática (Self-flushing end cap): Dispositivo que incluye un resorte que permite la salida del agua, al final de la cinta de goteo, cuando la presión del agua es menor a la presión umbral que el tapón puede contener.

Unidad de riego (Zone): Sección de un sistema de riego que puede ser operada simultáneamente por medio de una válvula de control.

Uniformidad de riego (Uniformity of water application): Medición del agua aplicada o almacenada en un suelo irrigado considerando la variación espacial, longitudinal a una hilera o transversal a varias hileras. La uniformidad de aplicación de agua usualmente es expresada en porcentaje, $100 \%$ representa una uniformidad perfecta. 
Válvula de control (Control valve): Dispositivo usado para controlar el flujo de agua. Las válvulas de control permiten o bloquean el paso del agua a las unidades de riego individuales.

Válvula de escape (Pressure-relief valve): Válvula que se abre para permitir la descarga de presión hacia la atmosfera cuando la presión en una tubería excede el nivel pre-determinado.

Velocidad del agua (Water velocity): Velocidad a la cual el agua se conduce en el interior de una tubería, usualmente expresada en $\mathrm{m} / \mathrm{s}$ (pies/segundo).

Zona radicular (Root zone): Profundidad y amplitud del perfil del suelo ocupado por las raíces de las plantas que están siendo irrigadas.

\section{Section 2. Drip Irrigation Technical Lexicon}

Acid (Ácido): A compound that releases $\mathrm{H}^{+}$ions when dissolved into solution. Compounds such as hydrochloric acid $(\mathrm{HCl})$ or acetic acid $\left(\mathrm{CH}_{3}-\mathrm{COOH}\right)$ are acids.

Acidification (Acidificación): The introduction of an acid [such as phosphoric, sulfuric or hydrochloric (muriatic) acid] into an irrigation system. This practice is mostly done in maintenance to improve the effectiveness of chlorination.

Algicide (Alguicida): A substance toxic to algae.

Anti-siphon device (Dispositivo anti-sifón) (see backflow-prevention device): A safety device used to prevent back flow of irrigation water into the water source by back-siphonage.

Application efficiency (Eficiencia de aplicación): The percentage of water applied by an irrigation system that is stored in the root zone and available for crop use.

Application rate (Caudal de goteo): The average rate at which water is applied by an irrigation system. For drip irrigation, rate is expressed as gallons/hour/100ft or gallons/ minute/emitter $(\mathrm{L} / \mathrm{h} / \mathrm{m}$ or $\mathrm{L} / \mathrm{min} /$ emitter$)$.

Backflow-prevention device (Dispositivo de prevención de retro-flujo) (see anti-siphon device): A device required by Florida law that prevents contaminated water from being sucked back into the water source should a reverse-flow situation occur.
Bactericide (Bactericida): A substance that kills bacteria.

Base (Base): A compound that produces $\mathrm{OH}^{-}$ions when dissolved into solution. Compounds such as potassium hydroxide $(\mathrm{KOH})$ or sodium hydroxide $(\mathrm{NaOH})$ are bases.

Best Management Practices (BMP) (Buenas Prácticas de Manejo, BPM): A set of cultural practices known to increase the efficiency of the irrigation and fertilization program while minimizing the environmental impact of production.

Certifying agency (Agencia de certificación): An independent, accredited third party that verifies that a certifiedorganic operation is compliant with the regulations described in the National Organic Standards as appropriate for their farming system.

Chelate (Quelato): A compound that binds polyvalent metals at two or more cation-exchange sites. Chelate is often a component of ready-to-use formulations for drip-irrigation cleaning. The use of synthetic chelates is not allowed in certified-organic production for cleaning drip-irrigation systems, but synthetic chelates may be used in certifiedorganic production to correct a documented micronutrient deficiency.

Chemigation (Quimirrigación): A general term referring to the application of water-soluble chemicals into the dripirrigation system. Chemigation includes (when allowable) the application of fertilizers, acids, chlorine and pesticides.

Chlorination (Cloración): The introduction of chlorine (at a calculated rate) into an irrigation system. Chlorination can use liquid sodium hypochlorite (household bleach) or chlorine gas. Some chlorinating agents are allowed in certified organic production.

Cleaning agent (Agente de limpieza): A substance used to remove dirt, filth and contaminants.

Control valve (Válvula de control): A device used to control the flow of water. Control valves turn on and off water to the individual zones.

Detergent (Detergente): A synthetic substance that is not soap and that is used to change the surface tension to remove oil and grease and other substances relatively insoluble in water. Detergents are not allowed in certified organic production. 
Disk filter (Filtro de discos): A stack of round, grooved disks used to filter water in a drip-irrigation system. As the size of the grooves decreases, the more the water is filtered. Each disk has grooves on both sides. Sediments and organic matter accumulate on the disks as water passes through the grooves. Disks are reusable. Once taken apart, they can be easily cleaned with pressured water and/or a detergent solution.

Drip irrigation (Riego por goteo): A method of irrigation using the slow application of water under low pressure through tube openings or attached devices just above, at or below the soil surface.

Electronic Database Information System (EDIS) (Sistema de Información electrónica de base de datos): The on-line database where the science-based, peer-reviewed and up-to-date recommendations of UF/IFAS Extension are accessible (https://edis.ifas.ufl.edu).

Emitter (Emisor o gotero): A dispensing device or opening in a micro-irrigation tube that regulates water application. An emitter creates a controlled flow expressed in gallons/ minute/emitter or gallons/100ft/h $(\mathrm{L} / \mathrm{min} / \mathrm{emitter}$ or $\mathrm{L} / \mathrm{m} / \mathrm{h})$.

Emitter spacing (Separación entre emisores): The distance between two consecutive emitters. Typical emitter spacings for vegetable crops are 4, 8, and 12 inches $(10,20$, and 30 $\mathrm{cm})$, and for blueberries is 18 inches $(45 \mathrm{~cm})$.

Evapotranspiration (ET) (Evapotranspiración): The combined losses of water by evaporation from the soil and transpiration from the plant.

Fertigation (Fertirrigación): The application of soluble fertilizer (plant nutrients) through a drip irrigation system. Fertigation is allowed in certified-organic systems provided the fertilizer sources used are allowed by NOP standards.

Field capacity (Capacidad de campo): The water content of the soil after all free water has been allowed to drain by gravity.

Filter (Filtro): A canister device containing a screen or a series of disks of a specified mesh or filled with a coarse solid medium and designed to catch solid particles large enough to clog emitters.

Fittings (Accesorios): The collective name of coupling and closure devices used to construct a drip system, which includes connectors, tees, elbows, goof plugs, and end caps.
Fittings may be of several types, including compression, barbed, or locking (spin or ring).

Flow rate (Caudal): The amount of water that moves through pipes in a given period of time. For micro-irrigation (drip irrigation), flow is expressed in gallons-per-hour (gph) or gallons-per-minute (gpm) (L/h or L/min).

Flow meter (Medidor de flujo): A device used to measure flow rate in a drip-irrigation system over the course of a crop cycle.

Goof plug (Tapón): An insertable cap used to plug holes in mainline and microtubes where drip devices have been removed or are no longer needed or when an accidental hole needs to be plugged.

Hole puncher (Perforador): A device that makes round holes in the pipes for connecting drip tape to laterals (available in different diameters).

Hydrochloric acid (HCl) (Acido Clorhídrico): An acid often used to lower the $\mathrm{pH}$ of water to increase the efficiency of chlorination. Use of $\mathrm{HCl}$ is prohibited in certified organic production.

Hypochlorous acid (HOCl) (Acido hipocloroso): The weak acid generated by chlorinating products. Hypochlorous acid destroys organic matter. Use of $\mathrm{HOCl}$ is restricted in organic production.

Irrigation schedule (Programación del riego): The watering plan and procedures that determine the proper amount of water to apply, the operating time, and the frequency of irrigation events.

Lateral line (Línea lateral): The actual drip line. The pipe on which or within which drippers are mounted or inserted.

Mainline (Línea principal): The tubing used in the drip system to conduct water from the source to the submainlines. It may be made of hard PVC or soft polyethylene material and comes in diameters ranging from 0.5-4 inches $(1.25-10 \mathrm{~cm})$.

Mazzei injector (Inyector Mazzei) (see venturi injector): A patented T-shaped, venturi-type injector that does not involve moving parts.

Media filter (Filtro de arena): A pressurized tank filled with fine gravel and sand. The sand is placed on top of the 
gravel. Sharp-edged sand or crushed rock are more efficient in catching soft algal tissue than round particles. Media filters should be used for filtering water that contains high levels of organic matter.

Micro-irrigation (Micro-irrigación): A synonym for drip irrigation.

Muriatic acid (Ácido muriático): Another name for hydrochloric acid $(\mathrm{HCl})$.

\section{National Organic Program (NOP) (Programa Nacional} de Producción Orgánica): The Federal Program created as a result of the Organic Foods Production Act of 1990 (title IX of the 1990 Farm Bill) and operated under the USDA Agriculture Marketing Service (http://www.ams.usda.gov/ nop/indexIE.htm).

Organic Materials Review Institute (OMRI) (Instituto de Revisión de Materiales Orgánicos): A national, nonprofit organization that determines which input products are compliant with the National Organic Program Standards (http://www.omri.org). The use of OMRI-listed products requires the approval of a certifying agency. Listing of a product on OMRI lists is not a guarantee of efficacy.

Overfertigating (Sobre-fertirrigar): Applying more fertilizer than the recommended rate. Overfertigating may result in nutrient leaching below the root zone.

Overwatering (Sobre-regar): Applying more water than necessary to meet the crop needs and/or applying water in excess of soil water-holding capacity. Overwatering potentially results in nutrient leaching below the root zone.

Part-per-million (ppm) (Partes por millón): The ratio of one in one million: $1 \mathrm{ppm}=1 / 1,000,000$. The "ppm" measurement may also represent concentrations: $1 \mathrm{ppm}=1$ $\mathrm{mg} / \mathrm{L} . ; 1 \%=10,000 \mathrm{ppm}$.

Peracetic acid (also known as peroxyacetic) (Ácido peracético, ácido conocido también como Peroxiacético): A mixture of acetic acid $\left(\mathrm{CH}_{3}-\mathrm{COOH}\right)$ and hydrogen peroxide $\left(\mathrm{H}_{2} \mathrm{O}_{2}\right)$ in an aqueous solution that can be used in certified organic production as a substitute for prohibited chlorination products.

Permanent wilting point (Punto de marchitez permanente): The water content of the soil in the plant root zone when the plant can no longer extract water from the soil. $\mathbf{p H}(\mathbf{p H})$ : A parameter expressing the amount of acidity (concentration of $\mathrm{H}^{+}$ions) in solution. It is represented by a number between 0 and 14 that is calculated as: $\mathrm{pH}=$ $-\log \left[\mathrm{H}^{+}\right] . \mathrm{pH}$ can be simply measured with a $\mathrm{pH}$-meter. A solution is acidic when $\mathrm{pH}<7$, neutral when $\mathrm{pH}=7$, and basic when $\mathrm{pH}>7$. $\mathrm{pH}$ affects the solubility and ionic forms in solution. $\mathrm{pH}$ is the single most important chemical parameter for water or soil.

Phosphoric acid ( $\mathrm{H}_{3} \mathrm{PO}_{4}$ ) (Acido Fosfórico): An acid often used to lower the $\mathrm{pH}$ of water so to increase the efficiency of chlorination. Use of phosphoric acid is prohibited in certified organic production.

Pressure (Presión): The "force" propelling water through pipes. Common static (nonflowing) pressure in irrigation systems is 20-70 psi (pounds-per-square-inch) (1.4-4.8 bar). Irrigation systems operate under dynamic (flowing) water pressure, which is reduced with elevation gain and friction loss caused by the water rubbing on the sides of pipes.

Pressure due to gravity (in pounds-per-square-inch or psi) (Presión por gravedad, en libras por pulgada cuadrada o psi): This measurement may be calculated as gain (downhill) or loss (uphill) by multiplying the height of the water column in feet by $0.433 \mathrm{psi} / \mathrm{ft}$ (in $\mathrm{m}$ by 0.0979 $\mathrm{bar} / \mathrm{m})$. For example, if a $200-\mathrm{ft}(60 \mathrm{~m})$ drip tape is laid on a field with a downhill slope of $3 \mathrm{ft} / 100 \mathrm{ft}(3 \%)[0.9 \mathrm{~m} / 30 \mathrm{~m}$ (3\%)], the gain in pressure due to gravity will be $200 \times 0.03$ x $0.433=2.59$ psi $(60 \times 0.03 \times 0.0979=0.18$ bar $)$.

Pressure loss (Pérdida de presión): The loss of water pressure under flow conditions caused by debris in a filter, friction in pipes and parts, and elevation changes.

Pressure rating (Presión nominal): The maximum pressure a pipe or drip-system component is able to handle without failing. For example, Class 160 PVC pipe refers to plastic irrigation pipe with a pressure rating of 160 pounds per square inch (psi) (11.0 bar). Aluminum irrigation pipe has a pressure rating of 145-150 psi (10.0-10.3 bar). These pressure ratings will normally be adequate for mainlines in drip-irrigation systems.

Pressure-relief valve (Válvula de escape): A valve that opens and discharges to the atmosphere to relieve the high pressure condition when pressure in a pipeline exceeds a pre-set point.

Pressure-compensating emitter (Emisor o gotero autocompensante de presión): An emitter designed to maintain 
a constant output (flow) over a wide range of operating pressures and elevations.

\section{Pressure-sensitive emitter (Emisor o gotero sensible a}

la presión): An emitter that releases more water at higher pressures and less at lower pressures, which are commonly used with long mainlines or terrain changes.

Pressure regulator (Regulador de presión): A device that reduces and maintains incoming water pressure for low pressure drip systems. Typical household water pressure is up to 5-60 psi (3.4-4.1 bar), while drip systems are designed to operate so not to exceed 8-12 psi (0.5-0.8 bar) in the drip tape. Due to friction losses, pressure in the delivery pipes may be 20-30 psi (1.4-2.0 bar), thereby requiring a pressure regulator. The important ratings of a pressure regulator are the diameter, the downstream pressure, and the maximum flow allowed by the pressure regulator.

Root zone (Zona radicular): The depth and width of soil profile occupied by the roots of the plants being irrigated.

Sand separator (Separador de arena): A device also called hydrocyclone that utilizes centrifugal force to separate sand and other heavy particles out of water. It is not a true filter, but could be considered a pre-filter.

Screen filter (Filtro de malla): A type of filter using a rigid screen to separate sand and other particulates out of irrigation water.

\section{Self-flushing end cap (Tapón de fin de línea con purga} automática): A spring-loaded device that lets water go out at the end of the drip tape when the water pressure is less than the threshold of the cap.

Sub-mainline (Línea secundaria): The tubing used to distribute water to a certain number of lateral lines. Also called secondary line.

Sulfuric acid $\left(\mathrm{H}_{2} \mathrm{SO}_{4}\right)$ (Acido sulfúrico): An acid often used to lower the $\mathrm{pH}$ of water so to increase the efficiency of chlorination. Use of sulfuric acid is prohibited in certified organic production.

Sulfur, powdered (Azufre en polvo): Elemental sulfur (S) in the yellow powder form is allowed in certified organic production. It is commonly used to decrease soil $\mathrm{pH}$, but this requires chemical conversion by soil microorganisms. Powdered sulfur should not be used for chlorination purposes.
Soap (Jabón): Alkaline salts of fatty acids used to remove hydrophilic particles.

Strong acid (Acido fuerte): An acid that is totally dissociated in water. Common strong acids are hydrochloric acid $(\mathrm{HCl})$ and sulfuric acid $\left(\mathrm{H}_{2} \mathrm{SO}_{4}\right)$.

Tape-to-lateral connector (Conector cinta-lateral): A device, sometimes called a barbed adapter, that is placed at the end of the drip tape (screw end) to connect it with the lateral (snap end).

Tape-to-tape connector (Conector cinta-cinta): A device used to repair or replace a leaking section of drip tape. The tape-to-tape connector allows two pieces of drip tape to be connected together.

Trickle irrigation (Riego por goteo): A synonym for drip irrigation.

Turbulent-flow emitter (Emisor o gotero de flujo turbulento): Emitters with a series of channels that force water to flow faster, thereby preventing particles from settling out and plugging the emitter.

Uniformity of water application (Uniformidad de riego): A measure of the spatial variability of water applied or stored in an irrigated field down a row and across several rows. Uniformity of water application is usually expressed as a percentage, $100 \%$ representing perfect uniformity.

Venturi injector (Inyector tipoVenturi): A tapered constriction which operates on the principle that a pressure drop accompanies the change in velocity of the water as it passes through the constriction. The pressure drop through a venturi must be sufficient to create a negative pressure (vacuum), relative to atmospheric pressure. Under these conditions, fluid from the tank will flow into the injector.

Water applied (Caudal real de riego): The amount of water actually applied during an irrigation cycle. For drip irrigation, it is expressed in gallons $/ 100$ feet of drip tape $(\mathrm{L} / \mathrm{m}$ of drip tape).

Water hardness (Dureza del agua): The sum of multivalent ions (such as calcium, magnesium, aluminum, or iron) in solution. Hardness is expressed in $\mathrm{mg} / \mathrm{L}$ or $\mathrm{ppm}$ of calcium carbonate equivalent, and its value is used to classify the water as soft $(0-20 \mathrm{mg} / \mathrm{L})$, moderately soft $(20-40 \mathrm{mg} / \mathrm{L})$, slightly hard (40-60 mg/L), moderately hard (60-80 mg/L), hard $(80-120 \mathrm{mg} / \mathrm{L})$, or very hard $(>120 \mathrm{mg} / \mathrm{L})$. 
Water alkalinity (Alcalinidad del agua): The ability of water to neutralize acids. Water alkalinity is based on the content of hydroxide $\left(\mathrm{OH}^{-}\right)$, carbonate $\left(\mathrm{CO}_{3}^{2-}\right)$ and bicarbonate $\left(\mathrm{HCO}_{3}^{-}\right)$ions.

Water velocity (Velocidad del agua): The speed at which water travels inside a pipe, usually expressed in feet/second $(\mathrm{m} / \mathrm{s})$.

Water hammer (Golpe de ariete): The pressure surge that occurs because of sudden stoppage or reduction in flow or because of a change in direction of flow. Water hammer may be reduced by slowly turning water on and also by an irrigation-system design in which water velocity is less than 5 feet $/$ second $(1.5 \mathrm{~m} / \mathrm{sec})$.

Weak acid (Ácido débil): An acid that is only partially dissociated in water. Common weak acids are phosphoric acid $\left(\mathrm{H}_{3} \mathrm{PO}_{4}\right)$, boric acid $\left(\mathrm{H}_{3} \mathrm{BO}_{3}\right)$, acetic acid $\left(\mathrm{CH}_{3}-\mathrm{COOH}\right)$, and citric acid $\left[\mathrm{COOH}-\mathrm{CH}_{2}-(\mathrm{COOH}-\mathrm{C}-\mathrm{OH})-\mathrm{CH}_{2}-\mathrm{COOH}\right]$.

Zone (Unidad de riego): A section of an irrigation system that can be operated at one time by means of a single control valve. 